\title{
Boundary value problems with four orders of Riemann-Liouville fractional derivatives
}

\author{
Somboon Niyom ${ }^{1}$, Sotiris K Ntouyas ${ }^{2,3}$, Sorasak Laoprasittichok ${ }^{4}$ and Jessada Tariboon ${ }^{4,5^{*}}$ (ID
}

${ }^{\text {*Correspondence: }}$

jessada.t@sci.kmutnb.ac.th

${ }^{4}$ Nonlinear Dynamic Analysis

Research Center, Department of

Mathematics, Faculty of Applied

Science, King Mongkut's University

of Technology North Bangkok,

Bangkok, 10800, Thailand

${ }^{5}$ Centre of Excellence in

Mathematics, CHE, Sri Ayutthaya

Rd., Bangkok, 10400, Thailand

Full list of author information is

available at the end of the article

\begin{abstract}
In this paper we study a new class of boundary value problems for fractional differential equations which contains Riemann-Liouville fractional derivatives of four orders, two in a fractional differential equation and two in boundary conditions. Our results are based on some classical fixed point theorems. Some illustrative examples are also included.
\end{abstract}

MSC: $34 \mathrm{~A} 08 ; 34 \mathrm{~A} 12$

Keywords: fractional differential equation; boundary value problem; existence; fixed point theorems

\section{Introduction}

In this paper, we investigate a boundary value problem which contains Riemann-Liouville fractional derivatives of four orders, two in a fractional differential equation and two in boundary conditions, of the form

$$
\left\{\begin{array}{l}
\left(\lambda D^{\alpha}+(1-\lambda) D^{\beta}\right) x(t)=f(t, x(t)), \quad t \in(0, T), \\
x(0)=0, \quad \mu D^{\gamma_{1}} x(T)+(1-\mu) D^{\gamma_{2}} x(T)=\gamma_{3},
\end{array}\right.
$$

where $D^{\phi}$ is the Riemann-Liouville fractional derivative of order $\phi \in\left\{\alpha, \beta, \gamma_{1}, \gamma_{2}\right\}$ such that $1<\alpha, \beta<2$ and $0<\gamma_{1}, \gamma_{2}<\alpha-\beta, \gamma_{3} \in \mathbb{R}$, the given constants $0<\lambda \leq 1,0 \leq \mu \leq 1$, and $f \in C([0, T] \times \mathbb{R}, \mathbb{R})$ is a continuous function.

Observe that the Riemann-Liouville fractional derivatives appearing in the differential equation and in the boundary conditions depend on the values of the constants $\lambda$ and $\mu$, respectively. If $\lambda=1$, then the first equation of (1.1) is reduced to a single order fractional derivative. Also in boundary conditions, the value of constant $\mu$ has an effect for fractional derivative of order $\gamma_{1}$ and $\gamma_{2}$. In applications, it seems that the values of $\lambda$ and $\mu$ can be interpreted as the adjustable instruments for a suitable real world phenomenon.

Fractional calculus has found numerous miscellaneous applications connected with real world problems as they appear in many fields of science and engineering, including fluid flow, signal and image processing, fractal theory, control theory, electromagnetic theory, fitting of experimental data, optics, potential theory, biology, chemistry, diffusion, and viscoelasticity. For a detailed account of applications and recent results on initial and boundary value problems of fractional differential equations, we refer the reader to [1-17] and the references therein.

(c) 2016 Niyom et al. This article is distributed under the terms of the Creative Commons Attribution 4.0 International License (http://creativecommons.org/licenses/by/4.0/), which permits unrestricted use, distribution, and reproduction in any medium, provided you give appropriate credit to the original author(s) and the source, provide a link to the Creative Commons license, and indicate if changes were made. 
The paper is organized as follows. In Section 2, we present the framework in which the boundary value problem (1.1), is formulated in a fixed point equation. Section 3 is devoted to the main results. Illustrative examples are also presented.

\section{Preliminaries}

In this section, we introduce some notations and definitions of fractional calculus $[1,2]$ and we present preliminary results needed in our proofs later.

Definition 2.1 The Riemann-Liouville fractional integral of order $\alpha>0$ of a function $g$ : $(0, \infty) \rightarrow \mathbb{R}$ is defined by

$$
I^{\alpha} g(t)=\int_{0}^{t} \frac{(t-s)^{\alpha-1}}{\Gamma(\alpha)} g(s) d s
$$

provided the right-hand side is point-wise defined on $(0, \infty)$, where $\Gamma$ is the Gamma function.

Definition 2.2 The Riemann-Liouville fractional derivative of order $\alpha>0$ of a continuous function $g:(0, \infty) \rightarrow \mathbb{R}$ is defined by

$$
D^{\alpha} g(t)=\frac{1}{\Gamma(n-\alpha)}\left(\frac{d}{d t}\right)^{n} \int_{0}^{t} \frac{g(s)}{(t-s)^{\alpha-n+1}} d s, \quad n-1<\alpha<n
$$

where $n=[\alpha]+1,[\alpha]$ denotes the integer part of real number $\alpha$, provided the right-hand side is point-wise defined on $(0, \infty)$.

From the definition of the Riemann-Liouville fractional derivative, we can obtain the following lemmas.

Lemma 2.1 (see [2]) Let $\alpha>0$ and $y \in C(0,1) \cap L(0,1)$. Then the fractional differential equation $D^{\alpha} y(t)=0$ has a unique solution

$$
y(t)=c_{1} t^{\alpha-1}+c_{2} t^{\alpha-2}+\cdots+c_{n} t^{\alpha-n},
$$

where $c_{i} \in \mathbb{R}, i=1,2, \ldots, n$, and $n-1<\alpha<n$.

Lemma 2.2 (see [2]) Let $\alpha>0$. Then for $y \in C(0,1) \cap L(0,1)$ we have

$$
I^{\alpha} D^{\alpha} y(t)=y(t)+c_{1} t^{\alpha-1}+c_{2} t^{\alpha-2}+\cdots+c_{n} t^{\alpha-n},
$$

where $c_{i} \in \mathbb{R}, i=1,2, \ldots, n$, and $n-1<\alpha<n$.

For convenience we use the constant

$$
\Lambda=\frac{\mu \Gamma(\alpha) T^{\alpha-\gamma_{1}-1}}{\Gamma\left(\alpha-\gamma_{1}\right)}+\frac{(1-\mu) \Gamma(\alpha) T^{\alpha-\gamma_{2}-1}}{\Gamma\left(\alpha-\gamma_{2}\right)} .
$$


Lemma 2.3 The boundary value problem (1.1) is equivalent to the following integral equation:

$$
\begin{aligned}
x(t)= & \frac{\lambda-1}{\lambda \Gamma(\alpha-\beta)} \int_{0}^{t}(t-s)^{\alpha-\beta-1} x(s) d s+\frac{1}{\lambda \Gamma(\alpha)} \int_{0}^{t}(t-s)^{\alpha-1} f(s, x(s)) d s \\
& +\frac{t^{\alpha-1}}{\Lambda}\left(\gamma_{3}-\frac{\mu(\lambda-1)}{\lambda \Gamma\left(\alpha-\beta-\gamma_{1}\right)} \int_{0}^{T}(T-s)^{\alpha-\beta-\gamma_{1}-1} x(s) d s\right. \\
& -\frac{\mu}{\lambda \Gamma\left(\alpha-\gamma_{1}\right)} \int_{0}^{T}(T-s)^{\alpha-\gamma_{1}-1} f(s, x(s)) d s \\
& -\frac{(1-\mu)(\lambda-1)}{\lambda \Gamma\left(\alpha-\beta-\gamma_{2}\right)} \int_{0}^{T}(T-s)^{\alpha-\beta-\gamma_{2}-1} x(s) d s \\
& \left.-\frac{1-\mu}{\lambda \Gamma\left(\alpha-\gamma_{2}\right)} \int_{0}^{T}(T-s)^{\alpha-\gamma_{2}-1} f(s, x(s)) d s\right), \quad t \in J:=[0, T],
\end{aligned}
$$

where the nonzero constant $\Lambda$ is defined by (2.1).

Proof From the first equation of (1.1), we have

$$
D^{\alpha} x(t)=\frac{\lambda-1}{\lambda} D^{\beta} x(t)+\frac{1}{\lambda} f(t, x(t)), \quad t \in J .
$$

Taking the Riemann-Liouville fractional integral of order $\alpha$ to both sides of (2.3), we get

$$
\begin{aligned}
x(t)= & \frac{\lambda-1}{\lambda \Gamma(\alpha-\beta)} \int_{0}^{t}(t-s)^{\alpha-\beta-1} x(s) d s+\frac{1}{\lambda \Gamma(\alpha)} \int_{0}^{t}(t-s)^{\alpha-1} f(s, x(s)) d s \\
& +C_{1} t^{\alpha-1}+C_{2} t^{\alpha-2}
\end{aligned}
$$

for $C_{1}, C_{2} \in \mathbb{R}$. Since $1<\alpha<2$, the first boundary condition of (1.1) implies that $C_{2}=0$. Hence

$$
\begin{aligned}
x(t)= & \frac{\lambda-1}{\lambda \Gamma(\alpha-\beta)} \int_{0}^{t}(t-s)^{\alpha-\beta-1} x(s) d s \\
& +\frac{1}{\lambda \Gamma(\alpha)} \int_{0}^{t}(t-s)^{\alpha-1} f(s, x(s)) d s+C_{1} t^{\alpha-1} .
\end{aligned}
$$

Applying the Riemann-Liouville fractional derivative of order $\psi \in\left\{\gamma_{1}, \gamma_{2}\right\}$ such that $0<$ $\psi<\alpha-\beta$ to (2.4), we have

$$
\begin{aligned}
D^{\psi} x(t)= & \frac{\lambda-1}{\lambda \Gamma(\alpha-\beta-\psi)} \int_{0}^{t}(t-s)^{\alpha-\beta-\psi-1} x(s) d s \\
& +\frac{1}{\lambda \Gamma(\alpha-\psi)} \int_{0}^{t}(t-s)^{\alpha-\psi-1} f(s, x(s)) d s+C_{1} \frac{\Gamma(\alpha)}{\Gamma(\alpha-\psi)} t^{\alpha-\psi-1} .
\end{aligned}
$$

Substituting the values $\psi=\gamma_{1}$ and $\psi=\gamma_{2}$ into the above relation and using the second condition of (1.1), we obtain

$$
\begin{aligned}
\gamma_{3}= & \frac{\mu(\lambda-1)}{\lambda \Gamma\left(\alpha-\beta-\gamma_{1}\right)} \int_{0}^{T}(T-s)^{\alpha-\beta-\gamma_{1}-1} x(s) d s \\
& +\frac{\mu}{\lambda \Gamma\left(\alpha-\gamma_{1}\right)} \int_{0}^{T}(T-s)^{\alpha-\gamma_{1}-1} f(s, x(s)) d s+\frac{\mu \Gamma(\alpha) T^{\alpha-\gamma_{1}-1}}{\Gamma\left(\alpha-\gamma_{1}\right)} C_{1}
\end{aligned}
$$




$$
\begin{aligned}
& +\frac{(1-\mu)(\lambda-1)}{\lambda \Gamma\left(\alpha-\beta-\gamma_{2}\right)} \int_{0}^{T}(T-s)^{\alpha-\beta-\gamma_{2}-1} x(s) d s \\
& +\frac{1-\mu}{\lambda \Gamma\left(\alpha-\gamma_{2}\right)} \int_{0}^{T}(T-s)^{\alpha-\gamma_{2}-1} f(s, x(s)) d s+\frac{(1-\mu) \Gamma(\alpha) T^{\alpha-\gamma_{2}-1}}{\Gamma\left(\alpha-\gamma_{2}\right)} C_{1}
\end{aligned}
$$

which leads to

$$
\begin{aligned}
C_{1}= & \frac{1}{\Lambda}\left[\gamma_{3}-\frac{\mu(\lambda-1)}{\lambda \Gamma\left(\alpha-\beta-\gamma_{1}\right)} \int_{0}^{T}(T-s)^{\alpha-\beta-\gamma_{1}-1} x(s) d s\right. \\
& -\frac{\mu}{\lambda \Gamma\left(\alpha-\gamma_{1}\right)} \int_{0}^{T}(T-s)^{\alpha-\gamma_{1}-1} f(s, x(s)) d s \\
& -\frac{(1-\mu)(\lambda-1)}{\lambda \Gamma\left(\alpha-\beta-\gamma_{2}\right)} \int_{0}^{T}(T-s)^{\alpha-\beta-\gamma_{2}-1} x(s) d s \\
& \left.-\frac{1-\mu}{\lambda \Gamma\left(\alpha-\gamma_{2}\right)} \int_{0}^{T}(T-s)^{\alpha-\gamma_{2}-1} f(s, x(s)) d s\right] .
\end{aligned}
$$

Substituting the value of the constant $C_{1}$ into (2.4), we deduce the integral equation (2.2). The converse follows by direct computation. This completes the proof.

\section{Main results}

Let $\mathcal{C}=C(J, \mathbb{R})$ denotes the Banach space of all continuous functions from $J:=[0, T]$ to $\mathbb{R}$ endowed with the usual sup-norm $\|u\|=\sup _{t \in J}|u(t)|$.

By Lemma 2.3, the boundary value problem (1.1) can be transformed to a fixed point problem $x=\mathcal{F} x$, where the operator $\mathcal{F}: \mathcal{C} \rightarrow \mathcal{C}$ is given by

$$
\begin{aligned}
\mathcal{F} x(t)= & \frac{\lambda-1}{\lambda \Gamma(\alpha-\beta)} \int_{0}^{t}(t-s)^{\alpha-\beta-1} x(s) d s+\frac{1}{\lambda \Gamma(\alpha)} \int_{0}^{t}(t-s)^{\alpha-1} f(s, x(s)) d s \\
& +\frac{t^{\alpha-1}}{\Lambda}\left(\gamma_{3}-\frac{\mu(\lambda-1)}{\lambda \Gamma\left(\alpha-\beta-\gamma_{1}\right)} \int_{0}^{T}(T-s)^{\alpha-\beta-\gamma_{1}-1} x(s) d s\right. \\
& -\frac{\mu}{\lambda \Gamma\left(\alpha-\gamma_{1}\right)} \int_{0}^{T}(T-s)^{\alpha-\gamma_{1}-1} f(s, x(s)) d s \\
& -\frac{(1-\mu)(\lambda-1)}{\lambda \Gamma\left(\alpha-\beta-\gamma_{2}\right)} \int_{0}^{T}(T-s)^{\alpha-\beta-\gamma_{2}-1} x(s) d s \\
& \left.-\frac{1-\mu}{\lambda \Gamma\left(\alpha-\gamma_{2}\right)} \int_{0}^{T}(T-s)^{\alpha-\gamma_{2}-1} f(s, x(s)) d s\right),
\end{aligned}
$$

where $\Lambda \neq 0$ is defined by (2.1). Observe that the boundary value problem (1.1) has a solution if and only if the associated fixed point problem $x=\mathcal{F} x$ has a fixed point.

For the sake of computational convenience, we use the notations

$$
\begin{aligned}
\Omega_{1}= & \frac{T^{\alpha-\beta}|\lambda-1|}{\lambda \Gamma(\alpha-\beta+1)}+\frac{T^{2 \alpha-\beta-\gamma_{1}-1} \mu|\lambda-1|}{\lambda \Lambda \Gamma\left(\alpha-\beta-\gamma_{1}+1\right)} \\
& +\frac{T^{2 \alpha-\beta-\gamma_{2}-1}(1-\mu)|\lambda-1|}{\lambda \Lambda \Gamma\left(\alpha-\beta-\gamma_{2}+1\right)}, \\
\Omega_{2}= & \frac{T^{\alpha}}{\lambda \Gamma(\alpha+1)}+\frac{T^{2 \alpha-\gamma_{1}-1} \mu}{\lambda \Lambda \Gamma\left(\alpha-\gamma_{1}+1\right)}+\frac{T^{2 \alpha-\gamma_{2}-1}(1-\mu)}{\lambda \Lambda \Gamma\left(\alpha-\gamma_{2}+1\right)} .
\end{aligned}
$$




\subsection{Existence and uniqueness result via Banach's fixed point theorem}

In the first result we prove an existence and uniqueness result by means of Banach's contraction mapping principle.

Theorem 3.1 Suppose that $f: J \times \mathbb{R} \rightarrow \mathbb{R}$ is a continuous function and satisfies the following assumption:

$\left(\mathrm{H}_{1}\right)$ there exists a constant $L>0$ such that $|f(t, x)-f(t, y)| \leq L|x-y|$, for each $t \in J$ and $x, y \in \mathbb{R}$.

If

$$
L \Omega_{2}+\Omega_{1}<1
$$

where $\Omega_{1}, \Omega_{2}$ are defined by (3.2) and (3.3), respectively, then the boundary value problem (1.1) has a unique solution on $J$.

Proof Setting $\sup _{t \in J}|f(t, 0)|=N<\infty$, and choosing

$$
R \geq \frac{\Lambda N \Omega_{2}+\left|\gamma_{3}\right| T^{\alpha-1}}{\Lambda\left(1-L \Omega_{2}-\Omega_{1}\right)}
$$

where $\Lambda$ is given by (2.1), as a first step, we show that $\mathcal{F} B_{R} \subset B_{R}$, where $B_{R}=\{x \in \mathcal{C}:\|x\| \leq$ $R$. For any $x \in B_{R}$, we have

$$
\begin{aligned}
|\mathcal{F} x(t)| \leq & \sup _{t \in J} \mid \frac{\lambda-1}{\lambda \Gamma(\alpha-\beta)} \int_{0}^{t}(t-s)^{\alpha-\beta-1} x(s) d s+\frac{1}{\lambda \Gamma(\alpha)} \int_{0}^{t}(t-s)^{\alpha-1} f(s, x(s)) d s \\
& +\frac{t^{\alpha-1}}{\Lambda}\left(\gamma_{3}-\frac{\mu(\lambda-1)}{\lambda \Gamma\left(\alpha-\beta-\gamma_{1}\right)} \int_{0}^{T}(T-s)^{\alpha-\beta-\gamma_{1}-1} x(s) d s\right. \\
& -\frac{\mu}{\lambda \Gamma\left(\alpha-\gamma_{1}\right)} \int_{0}^{T}(T-s)^{\alpha-\gamma_{1}-1} f(s, x(s)) d s \\
& -\frac{(1-\mu)(\lambda-1)}{\lambda \Gamma\left(\alpha-\beta-\gamma_{2}\right)} \int_{0}^{T}(T-s)^{\alpha-\beta-\gamma_{2}-1} x(s) d s \\
& \left.-\frac{1-\mu}{\lambda \Gamma\left(\alpha-\gamma_{2}\right)} \int_{0}^{T}(T-s)^{\alpha-\gamma_{2}-1} f(s, x(s)) d s\right) \mid \\
\leq & \frac{|\lambda-1|}{\lambda \Gamma(\alpha-\beta)} \int_{0}^{T}(T-s)^{\alpha-\beta-1}|x(s)| d s \\
& +\frac{1}{\lambda \Gamma(\alpha)} \int_{0}^{T}(T-s)^{\alpha-1}|f(s, x(s))-f(s, 0)|+|f(s, 0)| d s \\
& +\frac{T^{\alpha-1}\left(\left|\gamma_{3}\right|+\frac{\mu|\lambda-1|}{\lambda \Gamma\left(\alpha-\beta-\gamma_{1}\right)} \int_{0}^{T}(T-s)^{\alpha-\beta-\gamma_{1}-1}|x(s)| d s\right.}{\Lambda}\left(\frac{\mu}{\lambda \Gamma\left(\alpha-\gamma_{1}\right)} \int_{0}^{T}(T-s)^{\alpha-\gamma_{1}-1}|f(s, x(s))-f(s, 0)|+|f(s, 0)| d s\right. \\
& +\frac{(1-\mu)|\lambda-1|}{\lambda \Gamma\left(\alpha-\beta-\gamma_{2}\right)} \int_{0}^{T}(T-s)^{\alpha-\beta-\gamma_{2}-1}|x(s)| d s \\
& \left.+\frac{1-\mu}{\lambda \Gamma\left(\alpha-\gamma_{2}\right)} \int_{0}^{T}(T-s)^{\alpha-\gamma_{2}-1}|f(s, x(s))-f(s, 0)|+|f(s, 0)| d s\right) \\
& +\frac{1}{\lambda(s)}(s)
\end{aligned}
$$




$$
\begin{aligned}
\leq & (L\|x\|+N)\left[\frac{T^{\alpha}}{\lambda \Gamma(\alpha+1)}+\frac{T^{2 \alpha-\gamma_{1}-1} \mu}{\lambda \Lambda \Gamma\left(\alpha-\gamma_{1}+1\right)}+\frac{T^{2 \alpha-\gamma_{2}-1}(1-\mu)}{\lambda \Lambda \Gamma\left(\alpha-\gamma_{2}+1\right)}\right] \\
& +\|x\|\left[\frac{T^{\alpha-\beta}|\lambda-1|}{\lambda \Gamma(\alpha-\beta+1)}+\frac{T^{2 \alpha-\beta-\gamma_{1}-1} \mu|\lambda-1|}{\lambda \Lambda \Gamma\left(\alpha-\beta-\gamma_{1}+1\right)}\right. \\
& \left.+\frac{T^{2 \alpha-\beta-\gamma_{2}-1}(1-\mu)|\lambda-1|}{\lambda \Lambda \Gamma\left(\alpha-\beta-\gamma_{2}+1\right)}\right]+\frac{\left|\gamma_{3}\right| T^{\alpha-1}}{\Lambda} \\
= & \left(L \Omega_{2}+\Omega_{1}\right) R+N \Omega_{2}+\frac{\left|\gamma_{3}\right| T^{\alpha-1}}{\Lambda} \leq R .
\end{aligned}
$$

This means that $\|F x\| \leq R$, which leads to $\mathcal{F} B_{R} \subset B_{R}$.

Next, we let $x, y \in \mathcal{C}$. Then, for $t \in J$, we have

$$
\begin{aligned}
\mid \mathcal{F} x(t) & -\mathcal{F} y(t) \mid \\
\leq & \frac{|\lambda-1|}{\lambda \Gamma(\alpha-\beta)} \int_{0}^{T}(T-s)^{\alpha-\beta-1}|x(s)-y(s)| d s \\
& +\frac{1}{\lambda \Gamma(\alpha)} \int_{0}^{T}(T-s)^{\alpha-1}|f(s, x(s))-f(s, y(s))| d s \\
& +\frac{T^{\alpha-1}}{\Lambda}\left(\frac{\mu|\lambda-1|}{\lambda \Gamma\left(\alpha-\beta-\gamma_{1}\right)} \int_{0}^{T}(T-s)^{\alpha-\beta-\gamma_{1}-1}|x(s)-y(s)| d s\right. \\
& +\frac{\mu}{\lambda \Gamma\left(\alpha-\gamma_{1}\right)} \int_{0}^{T}(T-s)^{\alpha-\gamma_{1}-1}|f(s, x(s))-f(s, y(s))| d s \\
& +\frac{(1-\mu)|\lambda-1|}{\lambda \Gamma\left(\alpha-\beta-\gamma_{2}\right)} \int_{0}^{T}(T-s)^{\alpha-\beta-\gamma_{2}-1}|x(s)-y(s)| d s \\
& \left.+\frac{1-\mu}{\lambda \Gamma\left(\alpha-\gamma_{2}\right)} \int_{0}^{T}(T-s)^{\alpha-\gamma_{2}-1}|f(s, x(s))-f(s, y(s))| d s\right) \\
\leq & L\|x-y\|\left[\frac{T^{\alpha}}{\lambda \Gamma(\alpha+1)}+\frac{T^{2 \alpha-\gamma_{1}-1} \mu}{\lambda \Lambda \Gamma\left(\alpha-\gamma_{1}+1\right)}+\frac{T^{2 \alpha-\gamma_{2}-1}(1-\mu)}{\lambda \Lambda \Gamma\left(\alpha-\gamma_{2}+1\right)}\right] \\
& +\|x-y\|\left[\frac{T^{\alpha-\beta}|\lambda-1|}{\lambda \Gamma(\alpha-\beta+1)}+\frac{T^{2 \alpha-\beta-\gamma_{1}-1} \mu|\lambda-1|}{\lambda \Lambda \Gamma\left(\alpha-\beta-\gamma_{1}+1\right)}\right. \\
& \left.+\frac{T^{2 \alpha-\beta-\gamma_{2}-1}(1-\mu)|\lambda-1|}{\lambda \Lambda \Gamma\left(\alpha-\beta-\gamma_{2}+1\right)}\right] \\
= & \left(L \Omega_{2}+\Omega_{1}\right)\|x-y\|,
\end{aligned}
$$

which implies that $\|\mathcal{F} x-\mathcal{F} y\| \leq\left(L \Omega_{2}+\Omega_{1}\right)\|x-y\|$. Since $L \Omega_{2}+\Omega_{1}<1, \mathcal{F}$ is a contraction. Therefore, by the Banach contraction mapping principle, we see that $\mathcal{F}$ has a fixed point which is the unique solution of the boundary value problem (1.1). The proof is completed.

Example 3.1 Consider the following boundary value problem which contains RiemannLiouville fractional derivatives of two orders in a differential equation and the conditions:

$$
\left\{\begin{array}{l}
\left(\frac{26}{27} D^{17 / 9}+\frac{1}{27} D^{15 / 8}\right) x(t)=\frac{e^{-t}}{2(7-t)^{2}}\left(\frac{x^{2}(t)+2|x(t)|}{|x(t)|+1}\right), \quad t \in[0,3] \\
x(0)=0, \quad \frac{2}{67} D^{1 / 100} x(3)+\frac{65}{67} D^{1 / 101} x(3)=\frac{2}{9}
\end{array}\right.
$$


Here $\alpha=17 / 9, \beta=15 / 8, \lambda=26 / 27, \mu=2 / 67, \gamma_{1}=1 / 100, \gamma_{2}=1 / 101, \gamma_{3}=2 / 9, T=3$, and $f(t, x)=\left(e^{-t} / 2(7-t)^{2}\right)\left(\left(x^{2}+2|x|\right) /(|x|+1)\right)$. Since $|f(t, x)-f(t, y)| \leq(1 / 16)|x-y|$, then $\left(\mathrm{H}_{1}\right)$ is satisfied with $L=1 / 16$. By direct computation, we have $\Lambda \approx 2.63552 \neq 0, \Omega_{1} \approx 0.07837$, and $\Omega_{2} \approx 9.16679$. Thus $L \Omega_{2}+\Omega_{1} \approx 0.65129<1$. Hence, by Theorem 3.1, the problem (3.6) has a unique solution on $[0,3]$.

\subsection{Existence result via Krasnoselskii's fixed point theorem}

Next, we prove an existence result based on Krasnoselskii's fixed point theorem.

Theorem 3.2 (Krasnoselskii's fixed point theorem [18]) Let $M$ be a closed, bounded, convex, and nonempty subset of a Banach space $X$. Let $A, B$ be operators such that

(a) $A x+B y \in M$ where $x, y \in M$;

(b) $A$ is compact and continuous;

(c) $B$ is a contraction mapping.

Then there exists $z \in M$ such that $z=A z+B z$.

Theorem 3.3 Let $f: J \times \mathbb{R} \rightarrow \mathbb{R}$ be a continuous function satisfying $\left(\mathrm{H}_{1}\right)$ in Theorem 3.1. In addition, assume that:

$\left(\mathrm{H}_{2}\right)|f(t, x)| \leq v(t), \forall(t, x) \in J \times \mathbb{R}$ and $v \in C\left(J, \mathbb{R}^{+}\right)$.

Then the boundary value problem (1.1) has at least one solution on J, provided

$$
\Omega_{1}<1
$$

where $\Omega_{1}$ is defined by (3.2).

Proof Denoting $\|v\|=\sup _{t \in J}|v(t)|$, we consider the set $B_{r}=\{x \in \mathcal{C}:\|x\| \leq r\}$, where

$$
r \geq \frac{\Lambda\|v\| \Omega_{2}+\left|\gamma_{3}\right| T^{\alpha-1}}{\Lambda\left(1-\Omega_{1}\right)}
$$

and $\Lambda, \Omega_{1}$, and $\Omega_{2}$ are given by (2.1), (3.2), and (3.3), respectively.

For $t \in J$ let us introduce the operators $F_{1}$ and $F_{2}$ on $B_{r}$ as

$$
\begin{aligned}
F_{1} x(t)= & \frac{(\lambda-1)}{\lambda \Gamma(\alpha-\beta)} \int_{0}^{t}(t-s)^{\alpha-\beta-1} x(s) d s \\
& -\frac{t^{\alpha-1} \mu(\lambda-1)}{\lambda \Lambda \Gamma\left(\alpha-\beta-\gamma_{1}\right)} \int_{0}^{T}(T-s)^{\alpha-\beta-\gamma_{1}-1} x(s) d s \\
& -\frac{t^{\alpha-1}(1-\mu)(\lambda-1)}{\lambda \Lambda \Gamma\left(\alpha-\beta-\gamma_{2}\right)} \int_{0}^{T}(T-s)^{\alpha-\beta-\gamma_{2}-1} x(s) d s, \\
F_{2} x(t)= & \frac{1}{\lambda \Gamma(\alpha)} \int_{0}^{t}(t-s)^{\alpha-1} f(s, x(s)) d s \\
& +\frac{t^{\alpha-1}}{\Lambda}\left[\gamma_{3}-\frac{\mu}{\lambda \Gamma\left(\alpha-\gamma_{1}\right)} \int_{0}^{T}(T-s)^{\alpha-\gamma_{1}-1} f(s, x(s)) d s\right. \\
& \left.-\frac{(1-\mu)}{\lambda \Gamma\left(\alpha-\gamma_{2}\right)} \int_{0}^{T}(T-s)^{\alpha-\gamma_{2}-1} f(s, x(s)) d s\right] .
\end{aligned}
$$


To show that $F_{1} x+F_{2} y \in B_{r}$, we let $x, y \in B_{r}$. Then we have

$$
\begin{aligned}
\mid F_{1} x( & (t)+F_{2} y(t) \mid \\
\leq & \sup _{t \in[0, T]} \mid \frac{(\lambda-1)}{\lambda \Gamma(\alpha-\beta)} \int_{0}^{t}(t-s)^{\alpha-\beta-1} x(s) d s+\frac{1}{\lambda \Gamma(\alpha)} \int_{0}^{t}(t-s)^{\alpha-1} f(s, y(s)) d s \\
& -\frac{t^{\alpha-1} \mu(\lambda-1)}{\lambda \Lambda \Gamma\left(\alpha-\beta-\gamma_{1}\right)} \int_{0}^{T}(T-s)^{\alpha-\beta-\gamma_{1}-1} x(s) d s \\
& -\frac{t^{\alpha-1}(1-\mu)(\lambda-1)}{\lambda \Lambda \Gamma\left(\alpha-\beta-\gamma_{2}\right)} \int_{0}^{T}(T-s)^{\alpha-\beta-\gamma_{2}-1} x(s) d s \\
& +\frac{t^{\alpha-1}}{\Lambda}\left[\gamma_{3}-\frac{\mu}{\lambda \Gamma\left(\alpha-\gamma_{1}\right)} \int_{0}^{T}(T-s)^{\alpha-\beta-\gamma_{1}-1} f(s, y(s)) d s\right. \\
& \left.-\frac{(1-\mu)}{\lambda \Gamma\left(\alpha-\gamma_{2}\right)} \int_{0}^{T}(T-s)^{\alpha-\gamma_{2}-1} f(s, y(s)) d s\right] \mid \\
\leq & \|x\|\left[\frac{T^{\alpha-\beta}|\lambda-1|}{\lambda \Gamma(\alpha-\beta+1)}+\frac{T^{2 \alpha-\beta-\gamma_{1}-1} \mu|\lambda-1|}{\lambda \Lambda \Gamma\left(\alpha-\beta-\gamma_{1}+1\right)}\right. \\
& \left.+\frac{T^{2 \alpha-\beta-\gamma_{2}-1}(1-\mu)|\lambda-1|}{\lambda \Lambda \Gamma\left(\alpha-\beta-\gamma_{2}+1\right)}\right]+\frac{\left|\gamma_{3}\right| T^{\alpha-1}}{\Lambda} \\
& +\|v\|\left[\frac{T^{\alpha}}{\lambda \Gamma(\alpha+1)}+\frac{T^{2 \alpha-\gamma_{1}-1} \mu}{\lambda \Lambda \Gamma\left(\alpha-\gamma_{1}+1\right)}+\frac{T^{2 \alpha-\gamma_{2}-1}(1-\mu)}{\lambda \Lambda \Gamma\left(\alpha-\gamma_{2}+1\right)}\right] \\
\leq & r \Omega_{1}+\|v\| \Omega_{2}+\frac{\left|\gamma_{3}\right| T^{\alpha-1}}{\Lambda} \leq r .
\end{aligned}
$$

It follows that $F_{1} x+F_{2} y \in B_{r}$. This claim that the condition (a) of Theorem 3.2 holds. To prove that $F_{1}$ is a contraction mapping, for $x, y \in B_{r}$, we have

$$
\begin{aligned}
\mid F_{1} x(t) & -F_{1} y(t) \mid \\
\leq & \frac{|\lambda-1|}{\lambda \Gamma(\alpha-\beta)} \int_{0}^{T}(T-s)^{\alpha-\beta-1}|x(s)-y(s)| d s \\
& +\frac{T^{\alpha-1} \mu|\lambda-1|}{\lambda \Lambda \Gamma\left(\alpha-\beta-\gamma_{1}\right)} \int_{0}^{T}(T-s)^{\alpha-\beta-\gamma_{1}-1}|x(s)-y(s)| d s \\
& +\frac{T^{\alpha-1}(1-\mu)|\lambda-1|}{\lambda \Lambda \Gamma\left(\alpha-\beta-\gamma_{2}\right)} \int_{0}^{T}(T-s)^{\alpha-\beta-\gamma_{2}-1}|x(s)-y(s)| d s \\
\leq & \left\{\frac{T^{\alpha-\beta}|\lambda-1|}{\lambda \Gamma(\alpha-\beta+1)}+\frac{T^{2 \alpha-\beta-\gamma_{1}-1} \mu|\lambda-1|}{\lambda \Lambda \Gamma\left(\alpha-\beta-\gamma_{1}+1\right)}\right. \\
& \left.+\frac{T^{2 \alpha-\beta-\gamma_{2}-1}(1-\mu)|\lambda-1|}{\lambda \Lambda \Gamma\left(\alpha-\beta-\gamma_{2}+1\right)}\right\}\|x-y\| \\
= & \Omega_{1}\|x-y\|,
\end{aligned}
$$

which is a contraction, since $\Omega_{1}<1$. Therefore, the condition (c) of Theorem 3.2 is satisfied.

Using the continuity of the function $f$, we see that the operator $F_{2}$ is continuous. For $x \in B_{r}$, it follows that

$$
\left\|F_{2} x\right\| \leq\|v\|\left\{\frac{T^{\alpha}}{\lambda \Gamma(\alpha+1)}+\frac{T^{2 \alpha-\gamma_{1}-1} \mu}{\lambda \Lambda \Gamma\left(\alpha-\gamma_{1}+1\right)}+\frac{T^{2 \alpha-\gamma_{2}-1}(1-\mu)}{\lambda \Lambda \Gamma\left(\alpha-\gamma_{2}+1\right)}\right\}=\Omega_{2}\|v\|,
$$


which implies that the operator $F_{2}$ is uniformly bounded on $B_{r}$. Now we are going to prove that $F_{2}$ is equicontinuous. Setting $\sup _{t \in J, x \in \mathbb{R}}|f(t, x)|=\bar{f}$, for each $t_{1}, t_{2}$ such that $t_{2}>t_{1}$ and for $x \in B_{r}$, we have

$$
\begin{aligned}
\left|F_{2} x\left(t_{2}\right)-F_{2} x\left(t_{1}\right)\right| & \\
\leq & \mid \frac{1}{\lambda \Gamma(\alpha)}\left[\int_{0}^{t_{2}}\left(t_{2}-s\right)^{\alpha-1} f(s, x(s)) d s-\int_{0}^{t_{1}}\left(t_{1}-s\right)^{\alpha-1} f(s, x(s)) d s\right] \\
& +\frac{\left|t_{2}^{\alpha-1}-t_{1}^{\alpha-1}\right|}{\Lambda}\left[\left|\gamma_{3}\right|+\frac{\mu}{\lambda \Gamma\left(\alpha-\gamma_{1}\right)} \int_{0}^{T}(T-s)^{\alpha-\beta-\gamma_{1}-1} f(s, x(s)) d s\right. \\
& \left.+\frac{(1-\mu)}{\lambda \Gamma\left(\alpha-\gamma_{2}\right)} \int_{0}^{T}(T-s)^{\alpha-\gamma_{2}-1} f(s, x(s)) d s\right] \mid \\
\leq & \bar{f} \frac{1}{\lambda \Gamma(\alpha)}\left[\int_{0}^{t_{1}}\left[\left(t_{2}-s\right)^{\alpha-1}-\left(t_{1}-s\right)^{\alpha-1}\right] d s+\int_{t_{1}}^{t_{2}}\left(t_{1}-s\right)^{\alpha-1} d s\right] \\
& +\bar{f} \frac{\left|t_{2}^{\alpha-1}-t_{1}^{\alpha-1}\right|}{\Lambda}\left[\left|\gamma_{3}\right|+\frac{\mu}{\lambda \Gamma\left(\alpha-\gamma_{1}\right)} \int_{0}^{T}(T-s)^{\alpha-\beta-\gamma_{1}-1} d s\right. \\
& \left.+\frac{(1-\mu)}{\lambda \Gamma\left(\alpha-\gamma_{2}\right)} \int_{0}^{T}(T-s)^{\alpha-\gamma_{2}-1} d s\right] \\
\leq & \bar{f}\left\{\frac{2\left(t_{2}-t_{1}\right)^{\alpha}+\left|t_{2}^{\alpha}-t_{1}^{\alpha}\right|}{\lambda \Gamma(\alpha+1)}+\frac{\left|t_{2}^{\alpha-1}-t_{1}^{\alpha-1}\right|}{\Lambda}\left[\left|\gamma_{3}\right|+\frac{\mu T^{\alpha-\gamma_{1}}}{\lambda \Gamma\left(\alpha-\gamma_{1}+1\right)}\right.\right. \\
& \left.\left.+\frac{(1-\mu) T^{\alpha-\gamma_{2}}}{\lambda \Gamma\left(\alpha-\gamma_{2}+1\right)}\right]\right\},
\end{aligned}
$$

which is independent of $x$ and tends to zero as $t_{1} \rightarrow t_{2}$. Hence $F_{2}$ is equicontinuous. Therefore $F_{2}$ is relatively compact on $B_{r}$ and, by the Arzelá-Ascoli theorem, $F_{2}$ is compact on $B_{r}$. Thus the condition (b) of Theorem 3.2 is satisfied. Therefore all conditions of Theorem 3.2 are satisfied, and consequently, the boundary value problem (1.1) has at least one solution on $J$. This completes the proof.

Example 3.2 Consider the following boundary value problem which contains RiemannLiouville fractional derivatives of two orders in a differential equation and the conditions:

$$
\left\{\begin{array}{l}
\left(\frac{4}{5} D^{12 / 7}+\frac{1}{5} D^{11 / 9}\right) x(t)=\frac{\cos ^{2} t}{(11-t)^{2}}\left(\frac{|x(t)|}{|x(t)|+3}\right), \quad t \in[0,4] \\
x(0)=0, \quad \frac{6}{19} D^{1 / 4} x(4)+\frac{13}{19} D^{1 / 5} x(4)=\frac{3}{17} .
\end{array}\right.
$$

Here $\alpha=12 / 7, \beta=11 / 9, \lambda=4 / 5, \mu=6 / 19, \gamma_{1}=1 / 4, \gamma_{2}=1 / 5, \gamma_{3}=3 / 17, T=4$, and $f(t, x)=$ $\left(\cos ^{2} t /\left((11-t)^{2}\right)\right)((|x| /(|x|+3)))$. By direct computation, we have $\Lambda \approx 2.053184 \neq 0$ and $\Omega_{1} \approx 0.743699<1$. Clearly,

$$
\begin{aligned}
|f(t, x)| & =\left|\frac{\cos ^{2} t}{(11-t)^{2}}\left(\frac{|x|}{|x|+3}\right)\right| \\
& \leq \frac{\cos ^{2} t}{(11-t)^{2}} .
\end{aligned}
$$

Hence, by Theorem 3.3 the problem (3.9) has at least one solution on [0, 4]. 


\subsection{Existence result via Leray-Schauder's nonlinear alternative}

Our final existence result is based on Leray-Schauder's nonlinear alternative.

Theorem 3.4 (Nonlinear alternative for single valued maps [19]) Let E be a Banach space, $C$ a closed, convex subset of $E, U$ an open subset of $C$ and $0 \in U$. Suppose that $\mathcal{F}: \bar{U} \rightarrow C$ is a continuous, compact (that is, $\mathcal{F}(\bar{U})$ is a relatively compact subset of $C$ ) map. Then either

(i) $\mathcal{F}$ has a fixed point in $\bar{U}$, or

(ii) there is a $x \in \partial U$ (the boundary of $U$ in $C$ ) and $\lambda \in(0,1)$ with $x=\lambda \mathcal{F}(x)$.

Theorem 3.5 Suppose that $f: J \times \mathbb{R} \rightarrow \mathbb{R}$ is a continuous function and the following conditions hold:

$\left(\mathrm{H}_{3}\right)$ there exist a continuous nondecreasing function $\psi:[0, \infty) \rightarrow(0, \infty)$ and a function $\varphi \in C\left(J, \mathbb{R}^{+}\right)$such that

$$
|f(t, x)| \leq \varphi(t) \psi(\|x\|) \quad \text { for each }(t, x) \in J \times \mathbb{R}
$$

$\left(\mathrm{H}_{4}\right)$ there exists a constant $M>0$ such that

$$
\frac{M \Lambda}{M \Lambda \Omega_{1}+\psi(M)\|\varphi\| \Lambda \Omega_{2}+T^{\alpha-1}\left|\gamma_{3}\right|}>1
$$

where $\Lambda, \Omega_{1}, \Omega_{2}$ are defined by (2.1), (3.2) and (3.3), respectively.

Then the boundary value problem (1.1) has at least one solution on J.

Proof Consider the operator $\mathcal{F}$ defined by (3.1). We will show that the boundary value problem (1.1) has at least one solution on $J$. To accomplish this, firstly, we shall show that $\mathcal{F}$ maps bounded sets (balls) into bounded sets in $\mathcal{C}$. For a number $\rho>0$, let $B_{\rho}=\{x \in \mathcal{C}$ : $\|x\| \leq \rho\}$ be a bounded ball in $\mathcal{C}$. Then for $t \in J$ we have

$$
\begin{aligned}
|\mathcal{F} x(t)| \leq & \sup _{t \in J} \mid \frac{\lambda-1}{\lambda \Gamma(\alpha-\beta)} \int_{0}^{t}(t-s)^{\alpha-\beta-1} x(s) d s \\
& +\frac{1}{\lambda \Gamma(\alpha)} \int_{0}^{t}(t-s)^{\alpha-1} f(s, x(s)) d s \\
& +\frac{t^{\alpha-1}}{\Lambda}\left(\gamma_{3}-\frac{\mu(\lambda-1)}{\lambda \Gamma\left(\alpha-\beta-\gamma_{1}\right)} \int_{0}^{T}(T-s)^{\alpha-\beta-\gamma_{1}-1} x(s) d s\right. \\
& -\frac{\mu}{\lambda \Gamma\left(\alpha-\gamma_{1}\right)} \int_{0}^{T}(T-s)^{\alpha-\gamma_{1}-1} f(s, x(s)) d s \\
& -\frac{(1-\mu)(\lambda-1)}{\lambda \Gamma\left(\alpha-\beta-\gamma_{2}\right)} \int_{0}^{T}(T-s)^{\alpha-\beta-\gamma_{2}-1} x(s) d s \\
& \left.-\frac{1-\mu}{\lambda \Gamma\left(\alpha-\gamma_{2}\right)} \int_{0}^{T}(T-s)^{\alpha-\gamma_{2}-1} f(s, x(s)) d s\right) \mid \\
\leq & \psi(\|x\|)\|\varphi\|\left[\frac{T^{\alpha}}{\lambda \Gamma(\alpha+1)}+\frac{T^{2 \alpha-\gamma_{1}-1} \mu}{\lambda \Lambda \Gamma\left(\alpha-\gamma_{1}+1\right)}+\frac{T^{2 \alpha-\gamma_{2}-1}(1-\mu)}{\lambda \Lambda \Gamma\left(\alpha-\gamma_{2}+1\right)}\right] \\
& +\|x\|\left[\frac{T^{\alpha-\beta}|\lambda-1|}{\lambda \Gamma(\alpha-\beta+1)}+\frac{T^{2 \alpha-\beta-\gamma_{1}-1} \mu|\lambda-1|}{\lambda \Lambda \Gamma\left(\alpha-\beta-\gamma_{1}+1\right)}\right.
\end{aligned}
$$




$$
\begin{aligned}
& \left.+\frac{T^{2 \alpha-\beta-\gamma_{2}-1}(1-\mu)|\lambda-1|}{\lambda \Lambda \Gamma\left(\alpha-\beta-\gamma_{2}+1\right)}\right]+\frac{\left|\gamma_{3}\right| T^{\alpha-1}}{\Lambda} \\
= & \psi(\rho)\|\varphi\| \Omega_{2}+\rho \Omega_{1}+\frac{\left|\gamma_{3}\right| T^{\alpha-1}}{\Lambda}
\end{aligned}
$$

and, consequently,

$$
\|\mathcal{F} x\| \leq \psi(\rho)\|\varphi\| \Omega_{2}+\rho \Omega_{1}+\frac{\left|\gamma_{3}\right| T^{\alpha-1}}{\Lambda} .
$$

After that we will show that the operator $\mathcal{F}$ maps bounded sets into equicontinuous sets of $\mathcal{C}$. Let $t_{1}, t_{2} \in J$ such that $t_{1}<t_{2}$ and $x \in B_{\rho}$. Then we have

$$
\begin{aligned}
\left|\mathcal{F} x\left(t_{2}\right)-\mathcal{F} x\left(t_{1}\right)\right| & \leq|\lambda-1| \\
\leq & \left.\frac{\mid \lambda \Gamma(\alpha-\beta)}{t_{2}}\left(t_{2}-s\right)^{\alpha-\beta-1} x(s) d s-\int_{0}^{t_{1}}\left(t_{1}-s\right)^{\alpha-\beta-1} x(s) d s\right] \\
& +\frac{1}{\lambda \Gamma(\alpha)}\left[\int_{0}^{t_{2}}\left(t_{2}-s\right)^{\alpha-1} f(s, x(s)) d s-\int_{0}^{t_{1}}\left(t_{1}-s\right)^{\alpha-1} f(s, x(s)) d s\right] \\
& +\frac{\left|t_{2}^{\alpha-1}-t_{1}^{\alpha-1}\right|}{\Lambda}\left(\left|\gamma_{3}\right|+\frac{\mu|\lambda-1|}{\lambda \Gamma\left(\alpha-\beta-\gamma_{1}\right)} \int_{0}^{T}(T-s)^{\alpha-\beta-\gamma_{1}-1} x(s) d s\right. \\
& +\frac{\mu}{\lambda \Gamma\left(\alpha-\gamma_{1}\right)} \int_{0}^{T}(T-s)^{\alpha-\gamma_{1}-1} f(s, x(s)) d s \\
& +\frac{(1-\mu)|\lambda-1|}{\lambda \Gamma\left(\alpha-\beta-\gamma_{2}\right)} \int_{0}^{T}(T-s)^{\alpha-\beta-\gamma_{2}-1} x(s) d s \\
& \left.+\frac{1-\mu}{\lambda \Gamma\left(\alpha-\gamma_{2}\right)} \int_{0}^{T}(T-s)^{\alpha-\gamma_{2}-1} f(s, x(s)) d s\right) \\
\leq & \|x\|\left[\frac{\left[2\left(t_{2}-t_{1}\right)^{\alpha-\beta}+\mid t_{2}^{\alpha-\beta}-t_{1}^{\alpha-\beta}\right]|\lambda-1|}{\lambda \Gamma(\alpha-\beta+1)}+\frac{\left|t_{2}^{\alpha-1}-t_{1}^{\alpha-1}\right|}{\Lambda}\left(\frac{|\lambda-1| \mu T^{\alpha-\beta-\gamma_{1}}}{\lambda \Gamma\left(\alpha-\beta-\gamma_{1}+1\right)}\right.\right. \\
& \left.\left.+\frac{|\lambda-1|(1-\mu) T^{\alpha-\beta-\gamma_{2}}}{\lambda \Gamma\left(\alpha-\beta-\gamma_{2}+1\right)}\right)\right]+\frac{\left|t_{2}^{\alpha-1}-t_{1}^{\alpha-1}\right|\left|\gamma_{3}\right|}{\Lambda} \\
& +\bar{f}\left[\frac{2\left(t_{2}-t_{1}\right)^{\alpha-1}+\left|t_{2}^{\alpha-1}-t_{1}^{\alpha-1}\right|}{\lambda \Gamma(\alpha+1)}+\frac{\left|t_{2}^{\alpha-1}-t_{1}^{\alpha-1}\right|}{\Lambda}\left(\frac{\mu T^{\alpha-\gamma_{1}}}{\lambda \Gamma\left(\alpha-\gamma_{1}+1\right)}\right.\right. \\
& \left.\left.+\frac{(1-\mu) T^{\alpha-\gamma_{2}}}{\lambda \Gamma\left(\alpha-\gamma_{2}+1\right)}\right)\right] .
\end{aligned}
$$

As $t_{2}-t_{1} \rightarrow 0$, the right-hand side of the above inequality tends to zero independently of $x \in B_{\rho}$. Therefore, by the Arzelá-Ascoli theorem, the operator $\mathcal{F}: \mathcal{C} \rightarrow \mathcal{C}$ is completely continuous.

The result will follow from the Leray-Schauder nonlinear alternative (Theorem 3.4) once we have proved the boundedness of the set of the solutions to equations $x=\theta \mathcal{F} x$ for $\theta \in$ $(0,1)$.

Let $x$ be a solution. Then, for $t \in J$, and following similar computations to the first step, we have

$$
|x(t)| \leq \psi(\|x\|)\|\varphi\| \Omega_{2}+\|x\| \Omega_{1}+\frac{\left|\gamma_{3}\right| T^{\alpha-1}}{\Lambda},
$$


which leads to

$$
\frac{\|x\| \Lambda}{\|x\| \Lambda \Omega_{1}+\psi(\|x\|)\|\varphi\| \Lambda \Omega_{2}+T^{\alpha-1}\left|\gamma_{3}\right|} \leq 1
$$

In view of $\left(\mathrm{H}_{4}\right)$, there exists a constant $M$ such that $\|x\| \neq M$. Setting the set

$$
U=\{x \in \mathcal{C}:\|x\|<M\}
$$

we see that the operator $\mathcal{F}: \bar{U} \rightarrow \mathcal{C}$ is continuous and completely continuous. From the choice of $U$, there is no $x \in \partial U$ such that $x=\theta \mathcal{F} x$ for some $\theta \in(0,1)$. Consequently, by the nonlinear alternative of Leray-Schauder type, we see that the operator $\mathcal{F}$ has a fixed point $x \in \bar{U}$ which is a solution of the boundary value problem (1.1). This completes the proof.

Example 3.3 Consider the following boundary value problem which contains RiemannLiouville fractional derivatives of two orders in a differential equation and the conditions:

$$
\left\{\begin{array}{l}
\left(\frac{21}{23} D^{17 / 11}+\frac{2}{23} D^{13 / 9}\right) x(t)=\frac{1}{7(t+3)}\left(\frac{x^{2}(t)}{|x(t)|+2}+\frac{2|x(t)|}{|x(t)|+1}\right), \quad t \in[0,4] \\
x(0)=0, \quad \frac{7}{12} D^{3 / 100} x(4)+\frac{5}{12} D^{5 / 99} x(4)=\frac{2}{5}
\end{array}\right.
$$

Here $\alpha=17 / 11, \beta=13 / 9, \lambda=21 / 23, \mu=7 / 12, \gamma_{1}=3 / 100, \gamma_{2}=5 / 99, \gamma_{3}=2 / 5, T=4$ and $f(t, x)=(1 / 7(t+3))\left(\left(x^{2} /(|x|+2)\right)+(2|x| /(|x|+1))\right)$. By direct computation, we have $\Lambda \approx 2.024068 \neq 0, \Omega_{1} \approx 0.22817$, and $\Omega_{2} \approx 13.76475$. Clearly,

$$
\begin{aligned}
|f(t, x)| & =\left|\frac{1}{7(t+3)}\left(\frac{x^{2}}{|x|+2}+\frac{2|x|}{|x|+1}\right)\right| \\
& \leq \frac{1}{7(t+3)}(|x|+2) .
\end{aligned}
$$

Choosing $\varphi(t)=1 / 7(t+3)$ and $\psi(|x|)=|x|+2$, we can show that there exists $M>14.8763$ satisfying

$$
\frac{M \Lambda}{M \Lambda \Omega_{1}+\psi(M)\|\varphi\| \Lambda \Omega_{2}+T^{\alpha-1}\left|\gamma_{3}\right|}>1
$$

Hence, by Theorem 3.5, the problem (3.10) has at least one solution on [0,4].

\subsection{Special cases}

In this section, we discuss some special cases of the problem (1.1) which can be reduced to boundary value problems of Riemann-Liouville fractional derivatives with three orders. If $\lambda=1$, then the problem (1.1) is reduced to

$$
\left\{\begin{array}{l}
D^{\alpha} x(t)=f(t, x(t)), \quad t \in J, \\
x(0)=0, \quad \mu D^{\gamma_{1}} x(T)+(1-\mu) D^{\gamma_{2}} x(T)=\gamma_{3} .
\end{array}\right.
$$

Therefore, we have the following existence results. 
Corollary 3.1 Assume that the condition $\left(\mathrm{H}_{1}\right)$ holds. If $L \Omega_{2}<1$, then the boundary value problem (3.11) has a unique solution on $J$.

Corollary 3.2 Let the conditions $\left(\mathrm{H}_{1}\right)$ and $\left(\mathrm{H}_{2}\right)$ be satisfied. Then the boundary value problem (3.11) has at least one solution on J, provided $\Omega_{1}<1$.

Corollary 3.3 Suppose that the condition $\left(\mathrm{H}_{3}\right)$ is fulfilled. If there exists a positive constant M such that

$$
\frac{M \Lambda}{\psi(M)\|\varphi\| \Lambda \Omega_{2}+T^{\alpha-1}\left|\gamma_{3}\right|}>1
$$

then the boundary value problem (3.11) has at least one solution on J.

If $\mu=1$, then we have

$$
\left\{\begin{array}{l}
\left(\lambda D^{\alpha}+(1-\lambda) D^{\beta}\right) x(t)=f(t, x(t)), \quad t \in J, \\
x(0)=0, \quad D^{\gamma_{1}} x(T)=\gamma_{3},
\end{array}\right.
$$

and also a nonzero constant

$$
\Lambda=\frac{\Gamma(\alpha) T^{\alpha-\gamma_{1}-1}}{\Gamma\left(\alpha-\gamma_{1}\right)} .
$$

Corollary 3.4 Suppose that the condition $\left(\mathrm{H}_{1}\right)$ holds. If $L \Omega_{2}+\Omega_{1}<1$, where $\Omega_{1}, \Omega_{2}$ are defined by (3.2) and (3.3) and $\Lambda$ by (3.13), then the boundary value problem (3.12) has a unique solution on $J$.

Corollary 3.5 Assume that the conditions $\left(\mathrm{H}_{1}\right)$ and $\left(\mathrm{H}_{2}\right)$ are satisfied and suppose that $\Omega_{1}<1$ with $\Lambda$ defined by (3.13). Then the boundary value problem (3.12) has at least one solution on $J$.

Corollary 3.6 Assume that the conditions $\left(\mathrm{H}_{3}\right)$ and $\left(\mathrm{H}_{4}\right)$ are true with $\Lambda$ defined by (3.13). Then the boundary value problem (3.12) has at least one solution on $J$.

Competing interests

The authors declare that they have no competing interests.

Authors' contributions

All authors contributed equally in this article. They read and approved the final manuscript.

\section{Author details}

'Mathematics and Statistics Department, Faculty of Science, Nakhon Sawan Rajabhat University, Nakhon Sawan, 60000, Thailand. ${ }^{2}$ Department of Mathematics, University of loannina, loannina, 451 10, Greece. ${ }^{3}$ Nonlinear Analysis and Applied Mathematics (NAAM) - Research Group, Department of Mathematics, Faculty of Science, King Abdulaziz University, P.O. Box 80203, Jeddah, 21589, Saudi Arabia. ${ }^{4}$ Nonlinear Dynamic Analysis Research Center, Department of Mathematics, Faculty of Applied Science, King Mongkut's University of Technology North Bangkok, Bangkok, 10800, Thailand. ${ }^{5}$ Centre of Excellence in Mathematics, CHE, Sri Ayutthaya Rd., Bangkok, 10400, Thailand.

\section{Acknowledgements}

The authors thank the reviewers for their constructive comments, which led to the improvement of the original manuscript. This research is partially supported by the Centre of Excellence in Mathematics, the Commission on Higher Education, Thailand. 


\section{References}

1. Podlubny, I: Fractional Differential Equations. Academic Press, San Diego (1999)

2. Kilbas, AA, Srivastava, HM, Trujillo, JJ: Theory and Applications of Fractional Differential Equations. North-Holland Mathematics Studies, vol. 204. Elsevier, Amsterdam (2006)

3. Sabatier, J, Agrawal, OP, Machado, JAT (eds.): Advances in Fractional Calculus: Theoretical Developments and Applications in Physics and Engineering. Springer, Dordrecht (2007)

4. Ahmad, B, Ntouyas, SK, Alsaedi, A: New existence results for nonlinear fractional differential equations with three-point integral boundary conditions. Adv. Differ. Equ. 2011, Article ID 107384 (2011)

5. Alsaedi, A, Ntouyas, SK, Agarwal, RP, Ahmad, B: On Caputo type sequential fractional differential equations with nonlocal integral boundary conditions. Adv. Differ. Equ. 2015, 33 (2015)

6. Ahmad, B, Ntouyas, SK, Tariboon, J: Fractional differential equations with nonlocal integral and integer-fractional-order Neumann type boundary conditions. Mediterr. J. Math. (2015). doi:10.1007/s00009-015-0629-9

7. Bai, ZB, Sun, W: Existence and multiplicity of positive solutions for singular fractional boundary value problems. Comput. Math. Appl. 63, 1369-1381 (2012)

8. Su, Y, Feng, Z: Existence theory for an arbitrary order fractional differential equation with deviating argument. Acta Appl. Math. 118, 81-105 (2012)

9. Ahmad, B, Ntouyas, SK: Existence results for Caputo type sequential fractional differential inclusions with nonlocal integral boundary conditions. J. Appl. Math. Comput. 50, 157-174 (2016)

10. Alsaedi, A, Ntouyas, SK, Ahmad, B: New existence results for fractional integro-differential equations with nonlocal integral boundary conditions. Abstr. Appl. Anal. 2015, Article ID 205452 (2015)

11. Ntouyas, SK, Etemad, S, Tariboon, J: Existence of solutions for fractional differential inclusions with integral boundary conditions. Bound. Value Probl. 2015, 92 (2015)

12. Ahmad, B, Ntouyas, SK: Some fractional-order one-dimensional semi-linear problems under nonlocal integral boundary conditions. Rev. R. Acad. Cienc. Exactas Fís. Nat., Ser. A Mat. 110, 159-172 (2016)

13. Graef, JR, Kong, L, Wang, M: Existence and uniqueness of solutions for a fractional boundary value problem on a graph. Fract. Calc. Appl. Anal. 17, 499-510 (2014)

14. Tariboon, J, Ntouyas, SK, Thiramanus, P: Riemann-Liouville fractional differential equations with Hadamard fractional integral conditions. Int. J. Appl. Math. Stat. 54, 119-134 (2016)

15. Ahmad, B, Agarwal, RP: Some new versions of fractional boundary value problems with slit-strips conditions. Bound. Value Probl. 2014, 175 (2014)

16. Tariboon, J, Ntouyas, SK, Sudsutad, W: Fractional integral problems for fractional differential equations via Caputo derivative. Adv. Differ. Equ. 2014, 181 (2014)

17. Ntouyas, SK, Tariboon, J, Thaiprayoon, C: Nonlocal boundary value problems for Riemann-Liouville fractional differential inclusions with Hadamard fractional integral boundary conditions. Taiwan. J. Math. 20, 91-107 (2016)

18. Krasnoselskii, MA: Two remarks on the method of successive approximations. Usp. Mat. Nauk 10, 123-127 (1955)

19. Granas, A, Dugundji, J: Fixed Point Theory. Springer, New York (2005)

\section{Submit your manuscript to a SpringerOpen ${ }^{\circledR}$ journal and benefit from:}

- Convenient online submission

Rigorous peer review

- Immediate publication on acceptance

- Open access: articles freely available online

- High visibility within the field

- Retaining the copyright to your article 